\title{
Breve análisis acerca de las investigaciones en antropología sociocultural y folklore circumpuneñas. Definición de problemática y recientes aportes
}

\section{Introducción}

En este trabajo utilizamos una estrategia para el análisis de la actividad científica antropológica-social referida a la Subárea Circumpuneña. Esta estrategia consiste en la elaboración de un paradigma descriptivo constituido por cinco categorías u orientaciones de investigación, las cuales consideramos relevantes para abordar situaciones socioantropológicas significativas de acuerdo a la naturaleza holística de la disciplina.

Idealmente una investigación debería contener en su seno, o estar apoyada o integrada por las orientaciones incluidas en el paradigma, que presentamos en seguida (ver Figura 1), y pensamos nos va a servir para alcanzar los objetivos siguientes:

a) Dar una visión general del quehacer antropológico relativo al Norte Grande y principalmente de la Subárea Circumpuneña, durante las últimas cuatro décadas.

b) Motivar un enriquecimiento de los enfoques y facilitar la complementación de los vacíos bibliográficos que es seguro que existen.

c) A través de un inventario bibliográfico visualizar el desarrollo de las principales líneas de investigación y contrastarlas con el modelo propuesto para poder dar cuenta de los vacíos, y proponer las nuevas líneas que podrían desarrollarse.

d) Reunir en la última parte algunos trabajos recientes que presentan la característica de globalidad integral y que constituyen una situación promisoria para el estudio antropológico, aun

1 Departamento de Ciencias Sociológicas y Antropológicas, Facultad de Filosofía, Humanidades y Educación, Universidad de Chile. Av. Larraín 9925, Santiago, CHILE. cuando ellos no se refieren a trabajos propios de la subárea (p.e., Senri Ethnological Studies, Programa MAB, etc.).

e) Por último, tentativamente sugerir algunos obstáculos que han determinado un crecimiento inorgánico de las líneas de estudio analizadas.

Con toda seguridad nuestro esquema es incompleto en cuanto a representar la producción total de trabajos para la subárea, pero estimamos que su verdadero espíritu es más bien el de constituir una herramienta descriptiva de análisis.

\section{Paradigma descriptivo y de análisis}

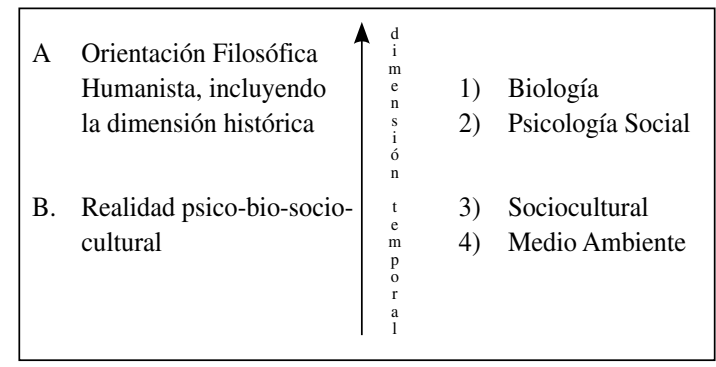

Figura 1. Paradigma descriptivo y de análisis.

Iniciamos este acápite con un esquema que refleja el carácter holístico de la disciplina antropológica y su vinculación con el ámbito filosófico-humanístico que es (A) y su proyección sobre su objeto de estudio que es (B). Este último está conformado por el aspecto psico-bio-socio-cultural de la sociedad y el propio ambiente físico, de donde se desprenden cuatro orientaciones antropológicas (1-4 de la columna derecha de la Figura 1, II a IV en su desarrollo, Cuadro 1).

\section{Orientación filosófico-humanística}

Esta orientación la consideramos como fundamento en el trasfondo de la investigación propiamente tal. Ella dice relación con el propio ethos (visión del mundo, ético, estético) del especialista que aborda 
la trama de relaciones bio-psicosocio-culturales y medioambiental que constituye el hombre y su comportamiento, objeto de estudio de nuestra disciplina. De acuerdo a la opinión o actitud que se tenga acerca del hombre, de su cultura y comportamiento se enfatizará como relevante una u otra de las orientaciones que hemos considerado (sin descartar por cierto la información que se tenga de las otras).

De este modo algunos investigadores explicarán, por ejemplo, el comportamiento del hombre mayormente sujeto a su condición biológica; otros realzarán los aspectos emocionales del comportamiento, $\mathrm{o}$ aspectos tecnológicos o también económicos. En síntesis, esta orientación, aun cuando privilegie (de acuerdo al propio investigador) un enfoque u orientación determinada como más relevante, reconoce la complementariedad de otros, es decir, siempre tiene una actitud propiamente humanista y está atribuyendo un sentido al estudio de investigación.

Esta orientación filosófico-humanista es importante en el contexto de descubrimiento, tanto en arqueología como en antropología social y física en el proceso de investigación. Y probablemente significativa también en el contexto de validación.

Conviene dejar constancia que esta orientación se debe preservar de una confusión corriente: la de no hacer distingos entre antropología filosófica (como región de la filosofía) y antropología (como ciencia social).

El título de libros y artículos (que hablan de antropología del arte, etc.) lleva a confusión al lego pues a veces son "filosofía del arte" (actitud humanista ante el arte) y no antropología social o cultural (como ciencia social) del arte.

\section{Orientación biológica}

Esta orientación está relacionada con el hombre concebido como un ente biológico en su más amplio sentido y comportándose, adaptándose, con o sin éxito, al ambiente, considerado también este último en forma amplia (como lo natural y lo sociocultural). Esta orientación involucra específicamente la biología cultural; los aspectos etnográficos y socioantropológicos que están entretejidos con aquellos netamente biológicos. Incluye tópicos como salud, salud mental y psiquiatría, aspectos psicosomáticos, transculturales, etc.
Orientación psicológico-social

Esta línea involucra cuatro subdimensiones:

a) La tradicional orientación de cultura y personalidad,

b) La orientación referente a las estructuras cognitivas diferenciales,

c) La etnociencia y por último;

d) Varias situaciones que podríamos ubicar dentro de un rubro denominado condicionamiento psicosocial diferencial. Por ejemplo, variabilidad del pensamiento concreto y abstracto (closed vs. open mind), tema importante para explicar aspectos de cambio, aceptación de innovaciones; diferencias mentales entre poblaciones o grupos rurales y urbanos, en la evolución psicológica, estructura rural, etc.

\section{Orientación sociocultural}

Esta dimensión comprende las estructuras culturales y sociales (sociológicas) y su interrelación, en el aspecto descriptivo y dinámico. Quedan comprendidos dentro de esta orientación los estudios de instituciones, familia, estratificación social, comunidades, religión, creencia, folklore. En este rubro se inscriben una antropología de la arquitectura, la proxémica, y estudio sobre dinámica en poblaciones, aculturación, etc.

\section{Orientación medioambiental}

Se refiere a todo lo que incluye el estudio de las unidades poblacionales y su forma de adaptación al medio ambiente físico. Especialmente considerando al todo como un sistema a través de su tecnología, formas económicas y captación de energía y/o información.

\section{Métodos y técnicas}

Aun cuando no podemos comprenderlo dentro de las orientaciones, no podemos dejar de referirnos al tema de métodos y técnicas en la antropología. Sobre esto parece que nosotros, aquí, deberíamos establecer un tópico de equilibrio entre lo cuantitativo y cualitativo. Pensamos que existen a veces ciertos peligros de que la disciplina antropológica pierda enfoques o técnicas etnográficas 


\begin{tabular}{|c|c|c|c|c|c|c|}
\hline \multirow{2}{*}{ Orientaciones } & \multicolumn{5}{|c|}{ Años } & \multirow{2}{*}{ Total } \\
\hline & 1940 & 1950 & 1960 & 1970 & 1980 & \\
\hline \multicolumn{7}{|l|}{ I. Orientación Filosófico-Humanística } \\
\hline 1.1. Estudios teóricos & & & & 1 & 2 & 3 \\
\hline 1.2. Estudios históricos & & & & & 1 & 1 \\
\hline \multicolumn{7}{|l|}{ II. Orientación biológica } \\
\hline \multicolumn{7}{|l|}{ 2.1. Antropología física } \\
\hline \multicolumn{7}{|l|}{ 2.2. Biología o sociocultural } \\
\hline \multicolumn{7}{|l|}{ 2.3. Salud } \\
\hline \multicolumn{7}{|l|}{ 2.4. Salud mental } \\
\hline \multicolumn{7}{|l|}{ 2.5. Psiquiatría transcultural } \\
\hline \multicolumn{7}{|l|}{ 2.6. Aspectos psicosomáticos } \\
\hline \multicolumn{7}{|l|}{ III. Línea de orientación psicológica, psicosocial } \\
\hline \multicolumn{7}{|l|}{ 3.1. Cultura y personalidad } \\
\hline 3.2. Estructuras cognitivas & & & & 1 & 1 & 2 \\
\hline \multicolumn{7}{|l|}{ 3.3. Etnociencia } \\
\hline \multicolumn{7}{|l|}{ 3.4. Condicionamiento de la inteligencia closed and open mind } \\
\hline \multicolumn{7}{|l|}{ IV. Línea sociocultural } \\
\hline 4.1. Estructuras culturales y sociales & & & & 2 & 2 & 4 \\
\hline \multicolumn{7}{|l|}{ 4.2. Instituciones, familia, estratificación social } \\
\hline 4.3. Comunidades, est. & 1 & & 2 & 11 & 2 & 16 \\
\hline 4.4. Folklore & & & 2 & 1 & & 3 \\
\hline 4.5. Religión, creencias, ritos & & 1 & 1 & 3 & & 5 \\
\hline \multicolumn{7}{|l|}{ 4.6. Proxémica } \\
\hline 4.7. Movilidad de poblaciones & & & & & 2 & 2 \\
\hline \multicolumn{7}{|l|}{ V. Sistemas medioambientales } \\
\hline Tecnología, economía & & & 2 & 1 & 2 & 5 \\
\hline Tecno ambiente & 1 & & & & 2 & 3 \\
\hline
\end{tabular}

Cuadro 1. Desarrollo de las líneas de orientaciones. Los estudios de antropología física no fueron tomados en cuenta porque los autores consideraron que ellos tienen que ver con el subsimposio paralelo a éste. Las cifras colocadas frente a cada rubro y bajo la columna de la década corresponden al respectivo número de trabajos.

clásicas (historias de vidas. Estudios de casos, observación-participante, etc.), frente a las promisorias técnicas cuantitativas de tipo estadístico y masificación de datos.

\section{Análisis de la muestra de investigación, aplicando el paradigma formulado}

Como lo expresamos anteriormente, la muestra analizada cuenta con 42 estudios que temporalmente se ubican en las últimas cuatro décadas. Ellos serán examinados cuantitativamente según períodos y también por tópicos de investigación
(Cuadro 2). Nuestra tarea en este subsimposio, en el que se nos pidió un plan de análisis, consistirá en llegar a reflexiones generales sobre el desarrollo de las líneas de orientación más que a un estudio monográfico de cada uno de los trabajos.

En general, en una dimensión cuantitativa creemos encontrar lo siguiente, de acuerdo al Cuadro 2, donde los estudios están ordenados según fecha de publicación; a la izquierda de cada trabajo figura un número que corresponde al estudio y que tiene una ordenación correlativa, y que se utiliza para referencia en el artículo. 


\begin{tabular}{|c|c|c|c|c|c|c|c|c|c|c|c|c|c|c|c|c|c|c|c|c|c|c|c|c|}
\hline 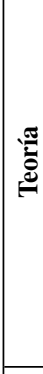 & 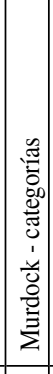 & & 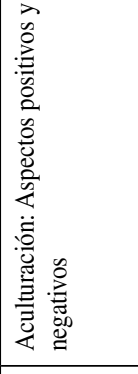 & 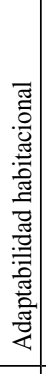 & & 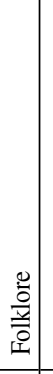 & & & & & 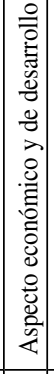 & 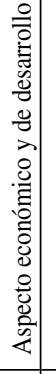 & & & & & & 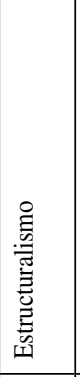 & & 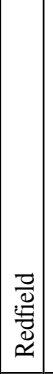 & 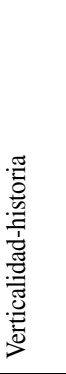 & & 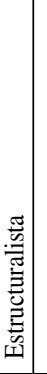 & \\
\hline 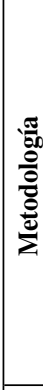 & 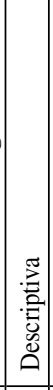 & 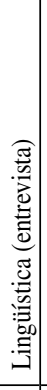 & 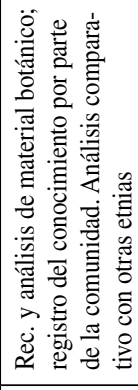 & 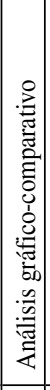 & 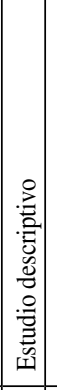 & 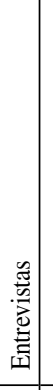 & 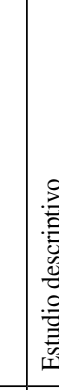 & 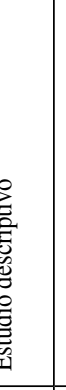 & 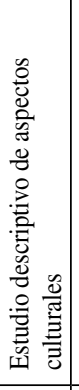 & 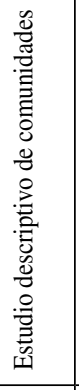 & 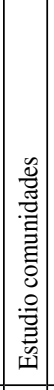 & 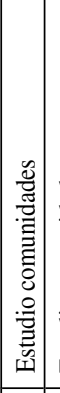 & 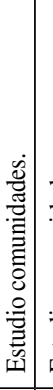 & 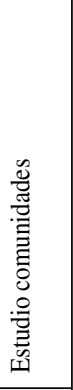 & 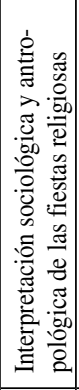 & 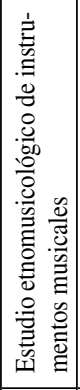 & 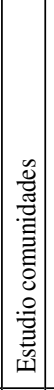 & 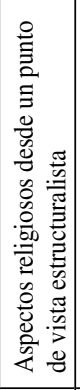 & 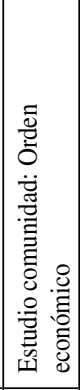 & 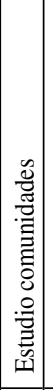 & 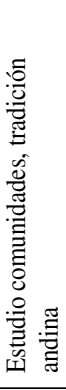 & 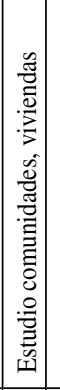 & 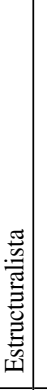 & 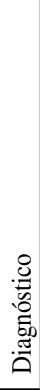 \\
\hline$\frac{\stackrel{0}{0}}{\stackrel{0}{0}}$ & 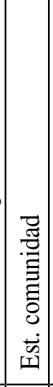 & 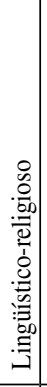 & 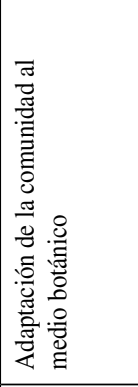 & 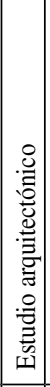 & 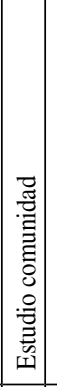 & 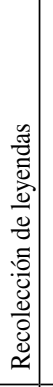 & & 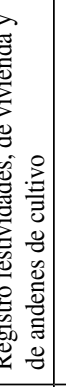 & 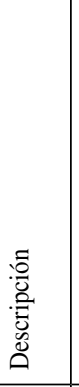 & 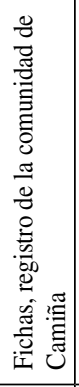 & 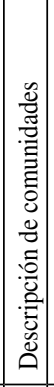 & 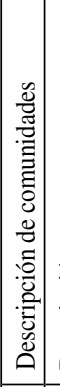 & 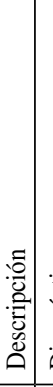 & 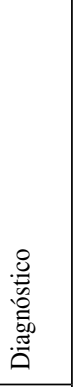 & & 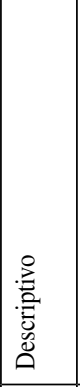 & 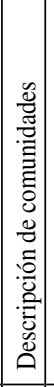 & 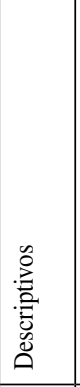 & & & & 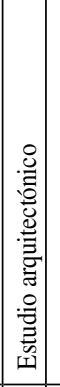 & 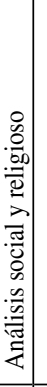 & \\
\hline 妾 & 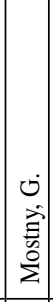 & 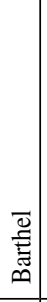 & 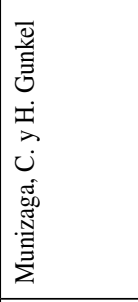 & 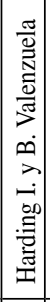 & 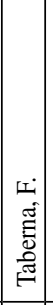 & $\begin{array}{l}\infty \\
\dot{0} \\
\dot{0} \\
\stackrel{0}{0} \\
\hat{\theta}\end{array}$ & 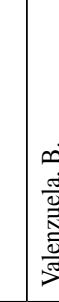 & 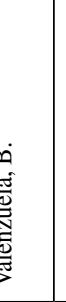 & $\begin{array}{l}> \\
\text { s. } \\
\text { b }\end{array}$ & 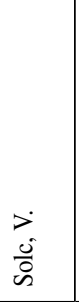 & 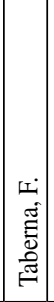 & 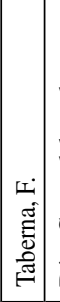 & 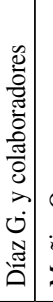 & 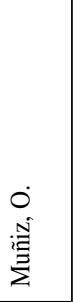 & 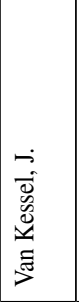 & 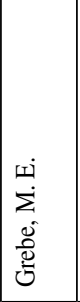 & 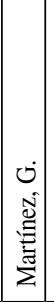 & 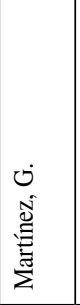 & 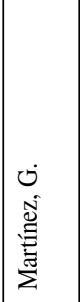 & 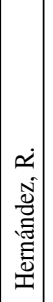 & 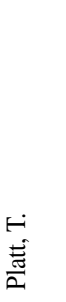 & 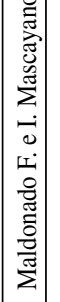 & 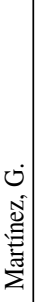 & $\begin{array}{l}0 \\
0 \\
0 \\
0 \\
0 \\
0 \\
0\end{array}$ \\
\hline 产 & : & 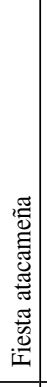 & 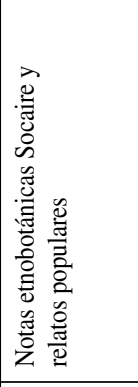 & 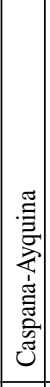 & 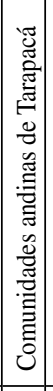 & 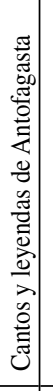 & 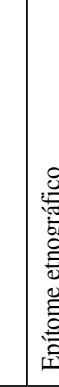 & 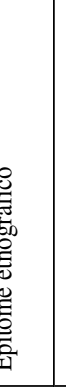 & 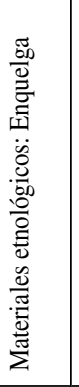 & 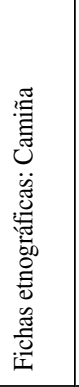 & 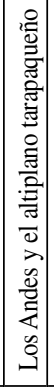 & $\mid$ & : & 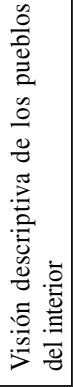 & 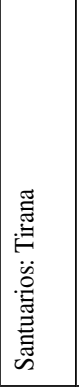 & 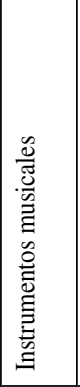 & 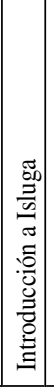 & 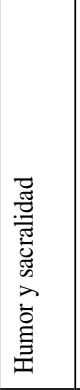 & 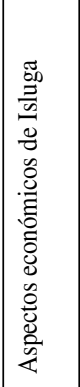 & $\begin{array}{l}\mathscr{0} \\
\overline{0} \\
\stackrel{0}{\varrho} \\
\end{array}$ & 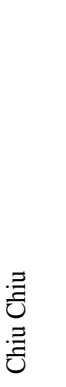 & 譬 & 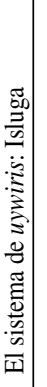 & 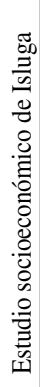 \\
\hline 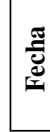 & $\begin{array}{l}q \\
\text { g } \\
= \\
=\end{array}$ & $\begin{array}{l}\tilde{n} \\
\approx \\
\approx\end{array}$ & 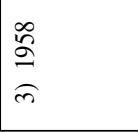 & 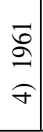 & $\begin{array}{l}\infty \\
\vdots \\
\approx \\
\approx\end{array} \mid$ & $\begin{array}{l}\frac{\pi}{2} \\
\stackrel{0}{6}\end{array}$ & 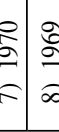 & 20 & $\begin{array}{l}\stackrel{\partial}{\varrho} \\
\text { ๙ }\end{array}$ & $\frac{\vec{a}}{\hat{\sigma}}$ & $\begin{array}{l}\vec{a} \\
\mathbf{a} \\
=\end{array}$ & $\begin{array}{l}\overrightarrow{5} \\
\widehat{\Xi} \\
\underline{\Xi}\end{array}$ & $\begin{array}{l}\vec{a} \\
\vec{\Omega} \\
\vec{\Omega}\end{array}$ & 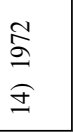 & $\frac{\tilde{2}}{\stackrel{2}{a}}$ & $\frac{\tilde{a}}{\hat{\sigma}}$ & 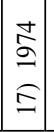 & $\begin{array}{l}\frac{1}{2} \\
\frac{\infty}{2} \\
\frac{\infty}{2}\end{array}$ & $\begin{array}{l}\stackrel{2}{a} \\
\stackrel{2}{\varrho}\end{array}$ & 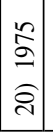 & 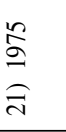 & & $\begin{array}{l}\stackrel{\vdots}{\Xi} \\
\widehat{\approx}\end{array}$ & 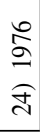 \\
\hline
\end{tabular}




\begin{tabular}{|c|c|c|c|c|c|c|c|c|c|c|c|c|c|c|c|c|}
\hline 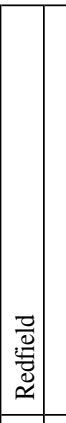 & & 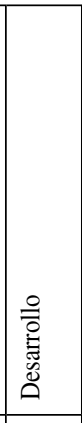 & & 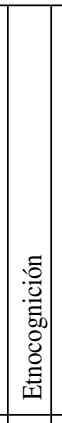 & 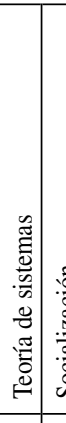 & 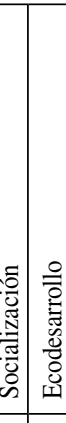 & 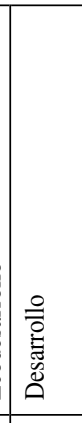 & & 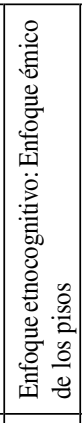 & 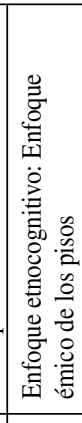 & & 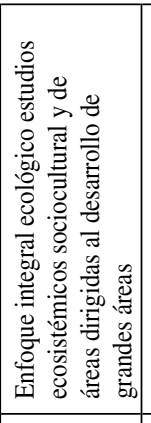 & & & & \begin{tabular}{|l} 
总 \\
总 \\
\end{tabular} \\
\hline 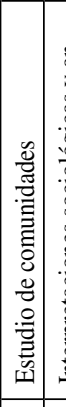 & 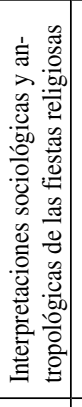 & 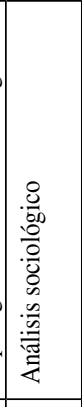 & 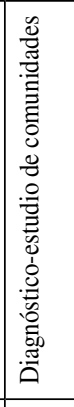 & & 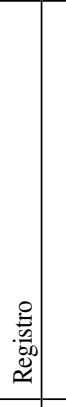 & 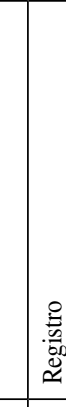 & & 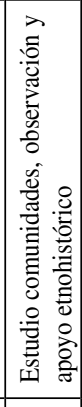 & 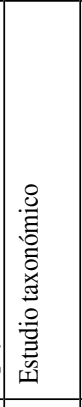 & 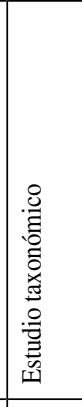 & 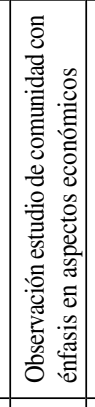 & & & & & 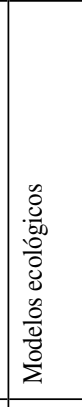 \\
\hline & & & & 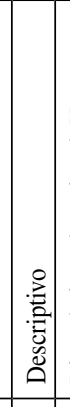 & 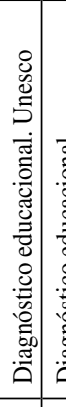 & & 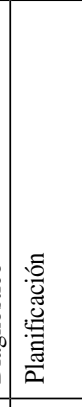 & 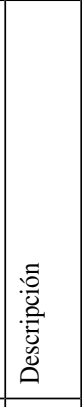 & 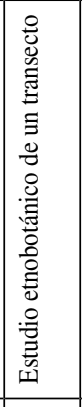 & 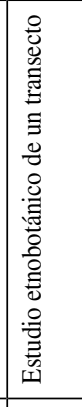 & & 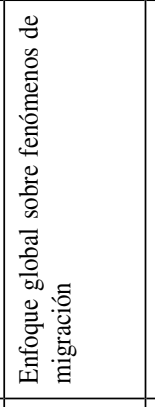 & 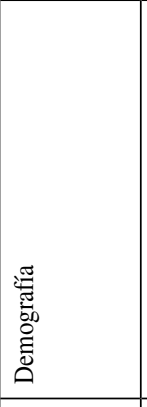 & 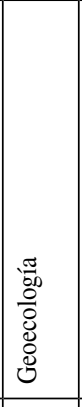 & 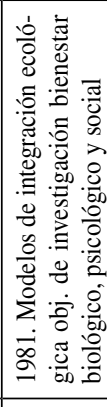 & 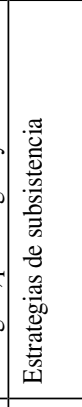 \\
\hline 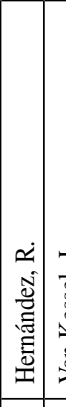 & 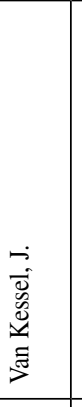 & 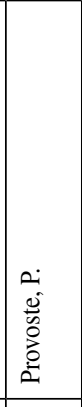 & 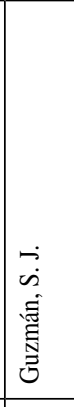 & 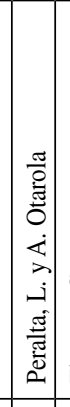 & 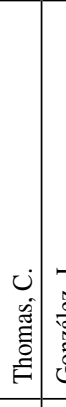 & 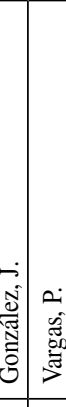 & 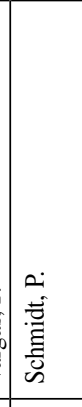 & 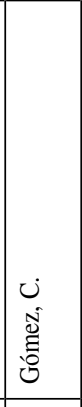 & 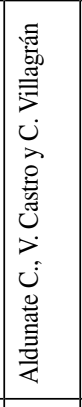 & 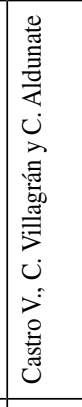 & 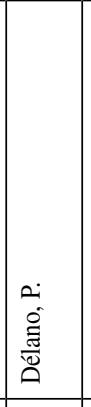 & 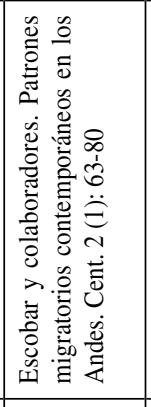 & 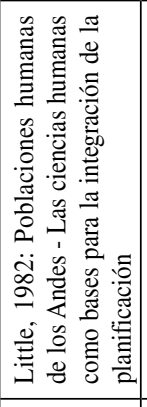 & 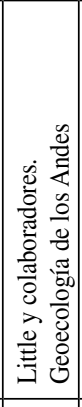 & 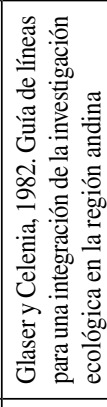 & 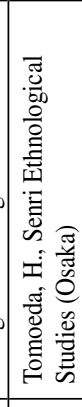 \\
\hline 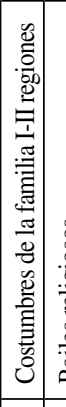 & 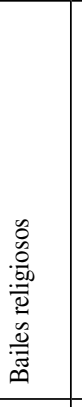 & 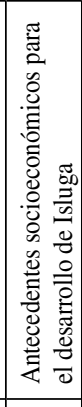 & 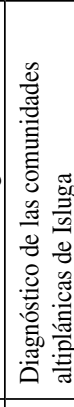 & 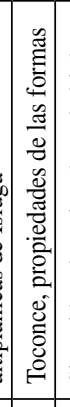 & 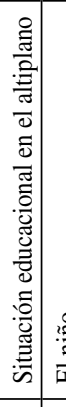 & 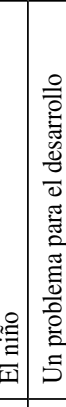 & 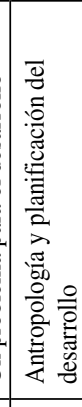 & 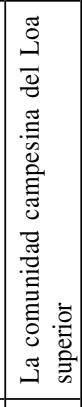 & 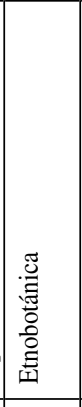 & 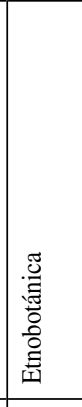 & 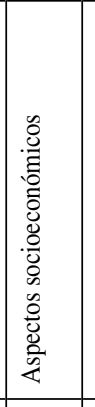 & 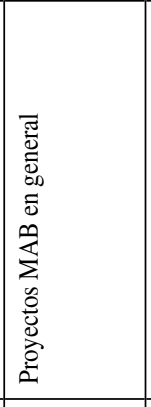 & & & & 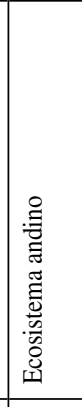 \\
\hline $\begin{array}{ll}0 \\
\vdots \\
\end{array}$ & 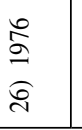 & 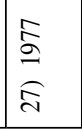 & 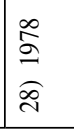 & $\left|\begin{array}{l}\infty \\
\vdots \\
\vdots \\
\hat{\mathrm{A}}\end{array}\right|$ & & 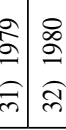 & 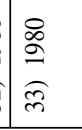 & 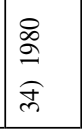 & $\begin{array}{l}\vec{\infty} \\
\stackrel{\vec{g}}{\vec{\rho}} \\
\vec{\rho}\end{array}$ & 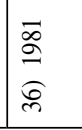 & 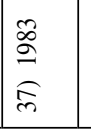 & 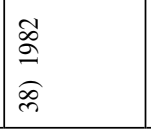 & 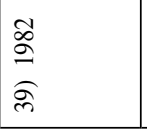 & 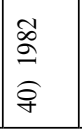 & 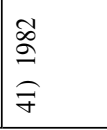 & $\widehat{f}$ \\
\hline
\end{tabular}

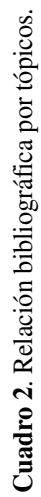


Una primera línea de orientación y la más desarrollada es aquella correspondiente a investigaciones socioculturales (IV). Dentro de las suborientaciones de esta línea, cuantitativamente, dominan aquellos estudios dedicados a comunidad (4.3): 16 trabajos, los cuales siguen en general un corte tradicional. Ellos comienzan tempranamente en la década de 1940 y alcanzan su máxima representación en la década de 1970, y prosiguen en la actual (Cuadro 2).

Una segunda suborientación que alcanza cierta importancia es la que dice relación con la religión y creencia (4.5), la cual en cierto modo es difícil separarla del folklore, pues las manifestaciones religiosas en su mayor parte dicen relación con expresiones de la religiosidad popular y la descripción de ciertos ritos. Sumando estas suborientaciones, habría 10 trabajos, los cuales surgen con una línea definida de investigaciones durante las décadas del 60 al 70 (ver Cuadro 2). En relación a la suborientación relativa a las estructuras culturales y sociales (4.1), la producción es escasa: tres trabajos, los cuales comienzan en la década del 70. Ciertas líneas dentro de esta misma orientación sociocultural como ser aquellas relacionadas al estudio de instituciones y familias, estratificación social, proxémica, no han constituido tópicos de interés para el estudio de los investigadores.

Una quinta orientación que nosotros hemos denominado sistemas medio ambientales (V), y que dice relación con aspectos de adaptación de la población a su ambiente físico está representada por ocho trabajos, los cuales en su mayoría dicen relación con el aspecto de cómo el hombre andino soluciona problemas como ser su habitación y el conocimiento de plantas y animales de su zona (trabajos de etnobotánica) y también modalidades zootécnicas (trabajos del Centro Isluga de la Universidad del Norte).

Con respecto a la primera orientación, relativa a la línea de estudios filosófico-humanista (I), podemos decir que muchos de los trabajos pertenecientes a otras orientaciones manejan esta línea pero más bien implícitamente. Faltan en cierto modo trabajos explícitos que desarrollen aspectos teóricos y consecuentemente manejen problemas e indicadores propios de tal enfoque. Sólo hemos encontrado dos de ellos que efectivamente explicitan ciertos aspectos referentes. Por ejemplo, uno de ellos desarrolla la tesis de cómo un sistema comunal indígena el cual se funda en una estructuración arcaica es, por influencia de sectores religiosos evangelizadores, desestabilizado y el otro de ellos utiliza las categorías manejadas por el enfoque de ecodesarrollo para diseñar ciertos cambios en la educación del altiplano tarapaqueño (Vargas 1980). Pero muchas de las categorías de orientación y las sublíneas que hemos bosquejado siguen como inexploradas. Por ejemplo, con relación a la orientación biológica, hay excelentes estudios de antropología física, los cuales no entramos a investigar porque ellos son conocidos y fueron tratados en el subsimposio de la especialidad. Pero aquellas líneas referentes a biología-sociocultural, salud mental, psiquiatría transcultural y aspectos psicosomáticos aún son líneas por explorar en el futuro, tanto en el norte en general como en la Subárea Circumpuneña en especial.

Línea de orientación psicológica (III): se encuentran tres trabajos en la línea etnobotánica y un trabajo en etnociencia presentado en este simposio.

De este crecimiento inorgánico del conocimiento antropológico acerca de la población circumpuneña de nuestro norte chileno, podemos extraer algunas conclusiones:

1. La falta de estudios que aborden el sentido humano, las implicaciones que fenómenos económicos, el conflicto cultural, la aculturación, adaptaciones biológicas, etc., pueden tener para el destino, del desarrollo integral del hombre, repercusiones negativas variadas. Una de ellas es que los estudios no presentan un sello que los haga candidatos a ingresar en el ámbito amplio del humanismo y las humanidades. Como consecuencia de lo anterior, que no establezcan puentes con los planes de educación, de desarrollo, y se queden marginados (a veces injustamente) de la plataforma sociocultural y política del país, lo que a la larga le resta amparo al desarrollo de la antropología, en todos sus campos. Es decir, que la investigación tienda a quedar cristalizada, pero "sin sentido".

2. Si bien es cierto podemos tener un cierto conocimiento de cómo son y cómo se organizan sus comunidades, cuál es su folklore y la persistencia de ciertos ritos como la limpia de canales, nada o muy poco podemos decir qué sabemos en aspectos tan importantes como su salud, mental y física, su formación moral, su personalidad, el condicionamiento de sus capacidades intelectuales, sólo por nombrar algunas líneas de conocimiento que nos faltan. De 
nuevo volvemos aquí al punto anterior: se requiere una revisión del sentido humano profundo que estas líneas tienen para comprender el desarrollo de la sociedad chilena, en regiones y comunidades.

3. Como consecuencia de lo antes dicho, podemos predecir, con cierto grado de certitud, que cualquier acción, plan o programa destinado a desarrollar la zona estará expuesto en un amplio margen a fracasar, no por el natural rechazo que toda población opone a los cambios, sino por la falta de conocimiento integral que tenemos sobre la zona.

\section{Recomendaciones}

1. Realizar estudios antropológicos que se apoyen, por lo menos, en un andamiaje global (que puede estar constituido por las orientaciones que hemos formulado). Que los estudios específicos de antropología sociocultural se ubiquen, coherentemente, dentro de tal estructura global. Los esfuerzos recientes expresados en estudios como Senri Ethnological Studies 1980 y Proyecto MAB 1982, se acercan a esta orientación holística propia de la antropología general.

El solo hecho de que en este congreso se hayan establecido subsimposios de antropología sociocultural, antropología física, etnohistoria, etc., muestra una clara y promisoria orientación para el desarrollo de la antropología general como gran disciplina integradora, dentro de la cual subcampos como los que antes se nombraron presentan un mismo rango o status científico. Con esto se contribuye a eliminar vanas discusiones que tienden a deteriorar uno u otro subcampo de la antropología general en su desarrollo.

2. Los trabajos de comunidad deben inscribirse dentro de contextos mayores, como la región y el país, lo que señala la necesidad de emplear métodos y técnicas de análisis regional. Debe continuarse la revisión de conceptos teóricos de comunidad, como unidad de análisis. Esto facilitará la decisión sobre las metodologías y técnicas a seguir. También permitirá tener a la vista, más claramente, las implicaciones prácticas (sobre desarrollo, modernización, educación, funciones de ellas dentro de sistemas regionales, etc.) de los estudios. Por ejemplo, urge hoy incorporar en los estudios de antropología social de comunidades, el nuevo elemento comuna-municipio, cuya estructura social, política, educacional y cuyas reales funciones manifiestas o latentes, todavía se desconocen.
3. Debe incrementarse el análisis filosófico, científicohumanista del área, dentro de los contextos andinos, chileno, americano, universal. Se corre el peligro de que tópicos tales como la aculturación, el conflicto de culturas diferentes, la evolución de la vida moral, religiosa, etc., no tengan una cabal comprensión, por la pérdida del constante y vivificador aliento de problematización a que estas importantes categorías son sometidas en el ámbito de la filosofía y en las orientaciones (a veces discrepantes) del humanismo. Tal problematización empuja a investigaciones científicas, exploraciones etnográficas, etc. El interés por desarrollar una etnografía del juego infantil en una comunidad, por ejemplo, podría casi carecer de sentido y podría perderse la pesquisa de elementos si no tenemos conciencia del efecto humanizador del juego. Este efecto y otras potencialidades del juego infantil para la formación moral intelectual y social, es un tema de constante y antigua revisión por parte de filósofos, cientistas sociales y naturales, pedagogos, materia que ya hemos considerado (Munizaga 1983). ${ }^{2}$

Advertimos, en este punto, la conveniencia de que el antropólogo tenga conciencia clara y no confunda su labor científica con su labor filosófica humanista, contribuyendo así al desarrollo de ambas y a su interfecundación.

4. Debe intensificarse la "cobertura" antropológicosocial de los múltiples hallazgos que surgen en materias relativas a las ciencias biológicas. Para ello es necesario que los fenómenos de variabilidad biológica, los de influencias ambientales como la altitud sobre el índice cefálico, o los procesos fisiológicos que trae el enrarecimiento de oxígeno, o las temperaturas extremas se liguen a la red de la etnografía material y sociocultural. Por ejemplo, a los tipos de vivienda. Pero, a su vez, el efecto que estos tipos de vivienda y su diseño tienden sobre el individuo, su mente, su cuerpo, su desarrollo intelectual y la estructura comunal o familiar. Hay que vincular el tópico de los problemas y variabilidad biológica con las costumbres, el "entrenamiento" social para la cultura (ya señalada por el pionero peruano Dr. Monge), con la dieta, con la medicina tradicional, etc. Incrementar, por ejemplo, los trabajos sobre percepción y autopercepción de los

\footnotetext{
2 Munizaga, C. Ms. Aproximación al estudio y organización de la actividad lúdica infantil urbana. A publicarse en Revista de Sociología y Antropología, Facultad de Filosofía, Humanidades y Educación, Universidad de Chile, Santiago.
} 
tipos físicos (citados por J. Munizaga en este simposio) con los problemas de endogamia, con los de conflicto y desajuste con los grupos "no indígenas" de las ciudades chilenas del Pacífico norte; con los problemas de antropología psicológica relativos a la autofirmación, deterioro y aun perturbaciones en la construcción del yo.

5. Finalmente, en lo relativo a métodos y técnicas debemos ser cautos para que los métodos cualitativos no sucumban frente a la necesaria y bienvenida cuantificación. Porque hay que recordar que una posición fundamental del antropólogo es la de ser etnógrafo, el que está siempre esperando que un dato, escaso, a veces único, en apariencia irrelevante y que solo se obtiene en la observación directa, puede dar nuevas pistas. Por eso es obvio recomendar que se mantenga un equilibrio entre los dos tipos de procedimiento. Que se tomen concienzudamente decisiones, cuando todo hace pensar que hay que renunciar a una hermosa y sofisticada cuantificación, en beneficio a una visión etnográfica que podría perderse. Curiosamente, a veces esta decisión por lo etnográfico, con plena conciencia y explícitamente la han tomado grandes sociólogos como Goffman (1970), en el estudio de instituciones "amuralladas" como las cárceles, los campos de prisioneros, los conventos, los hospitales psiquiátricos, los colegios de internos, etc.

Agradecimiento A M. Antonieta Benavente, quien colaboró en la realización de este resumen, en lo que respecta a la recolección y ordenamiento de la bibliografía.

\section{REFERENCIAS CITADAS}

ALDUNATE, C., V. CASTRO y C. VILLAGRAN, 1983. Etnobotánica. Boletín del Museo Nacional de Historia Natural p. 183-223.

ALVARADO, L., 1970. La vida rural en el altiplano chileno. Icira (Base de diagnóstico estructura agraria. Altiplano chileno).

CASTRO, V. y C. VILLAGRAN, 1982. Estudio etnobotánico en la precordillera y altiplano de los Andes del norte de Chile. En Etnobotánica de Chile, Bustos y Veloso (Eds.). Volumen de síntesis. Proyecto MAB-6 (2) Unesco, Montevideo.

CASTRO, M., 1981Ms. Estrategias socioculturales de subsistencia en las comunidades aymara altoandinas, en el interior de la Provincia de Arica.

DELANO, P., 1982. Aspectos socioeconómicos de una comunidad del Norte Grande: Caspana. Tesis de Grado. Universidad de Chile, Santiago.

DIAZ, G. y otros, 1971. Caspana: Una comunidad. Tesis Servicio Social, Universidad de Chile, Antofagasta.

GOFFMAN, E., 1970 Internados. Amorrortu Editores, Buenos Aires.

GOMEZ, C., 1980. La comunidad campesina indígena del Loa Superior. Tesis de Grado. Universidad de Chile, Santiago.

GONZALEZ, I., 1979. El niño aymara: Socialización y desocialización. Tambo 1 ( 3).

GREBE, M. E., 1973. Instrumentos musicales del Norte Grande de Chile. Congreso del Hombre Andino, Iquique.

1980. Generative models, simbolic structures and acculturation in the panpipe music of the aymara of Tarapacá, Chile. Tesis Doctoral. The Queens University of Belfast, Belfast.
GUZMAN, S. J., 1978. Elementos de diagnósticos de las comunidades altiplánicas de Isluga. Inconor Depto. Agrícola Iqq'Mimco, Iquique.

HARDING, J., 1961. Caspana-Ayquina. Tesis de Grado. Escuela de Arquitectura, Universidad de Chile, Santiago.

HERNANDEZ, A., R. POBLETE, y D. QUIROZ, 1975. Toconce, la vigencia de la comunidad tradicional. Revista de Antropología, Nueva Epoca 2.

HERNANDEZ, R., 1976. Costumbres de la familia y su planificación en las comunidades de la I y II Región. Publicación ocasional del Departamento del Antropología y Arqueología, Universidad de Chile.

MALDONADO, F. y I. MASCAYANO, 1975Ms. Ayquina: Un pueblo precordillerano andino. Seminario de Título. Escuela de Arquitectura, Universidad de Chile, Santiago.

MARTINEZ, G., 1974. Introducción a Isluga. Universidad de Chile. Iquique. Características de orden antropológico y socioeconómico de Isluga, I Región. Norte Grande I: $3-4$.

1976. El sistema de los uywiris de Isluga. Homenaje al Dr. Gustavo Le Paige s. j. Universidad del Norte, Antofagasta.

1974. Humor y sacralidad en el mundo autóctono andino. Universidad de Chile, Departamento de Investigaciones, Iquique.

MILLONES, L. y H. TOMOEDA, 1982. El hombre y su ambiente en los Andes Centrales. Senri Ethnological Studies, Japan National Museum of Ethnology 2, Osaka.

MOSTNY, G., y otros, 1940. Peine, un pueblo atacameño. Ministerio de Educación. Santiago. 
MUNIZAGA, C. y H. GUNCKEL, 1958. Notas etnobotánicas de Socaire. Universidad de Chile, CEA 5. Santiago.

MUÑIZ, O., 1972. Visión descriptiva de los pueblos del interior de la Provincia de Antofagasta. Odeplan Macrozona Norte, Santiago.

PERALTA, L. y A. OTAROLA, 1978Ms. Toconce, propiedades de las formas a través de la percepción. Seminario de Título. Escuela de Arquitectura, Universidad de Chile, Santiago.

PLATT, T., 1975. Reseña bibliográfica sobre el artículo de Roberto Hernández: Chiu Chiu: La desintegración de la comunidad tradicional. Chungara 5: 153.

PROVOSTE, P., 1976. Antecedentes de la estructura socioeconómica de Isluga. Universidad del Norte. Centro Isluga de Investigaciones Andinas, Iquique.

1977. Antecedentes socioeconómicos para el desarrollo del sector Isluga, Iquique. Universidad del Norte, Centro Isluga de Investigaciones Andinas, Iquique.

SCHMIDT, P., 1980. Antropología y planificación del desarrollo. Tesis de Grado en Antropología, Universidad de Chile, Santiago.

SOLC, V., 1969Ms. Materiales etnológicos recogidos en Enquelga. Universidad de Chile, Departamento de Relaciones Internacionales, Iquique.

1971Ms. Fichas etnográficas de Camiña. Universidad de Chile, Iquique.

SOTO, F. Tradición religiosa y Ayquina. Revista de Carabineros 188: 58-59.

TABERNA, F., 1968. Algo sobre las comunidades andinas de Tarapacá. Estudios Regionales 1: 7-17.
_ 1971a. Los Andes y el altiplano tarapaqueño. Publicación del Departamento de Ciencias Sociales de la Universidad de Chile.

197Ib. Isluga. Noticiero de la Universidad de Chile.

THOMAS, C., 1978. Situación educacional en el altiplano chileno. En Oficina Regional de Educación de la Unesco para América Latina y el Caribe. Unesco, Santiago.

TOLOSA, B., 1967. Cantos y leyendas regionales. Antofagasta.

TOMOEDA, H. (Ed.), 1980. Ponencias presentadas en el $4^{\circ}$ Simposio Internacional, Museo Nacional de Etnología. Senri Ethnological Studies.

1982. Folklore andino y mitología amazónica: Las plantas culturales y la muerte en el pensamiento andino. Senri Ethnological Studies 10: 275-307.

VALENZUELA, B., 1969. Epítome etnográfico de la cuenca del río Salado. Provincia de Antofagasta. Boletín de Prehistoria Chilena 2 (2-3).

VAN KESSEL, J., 1976. Los bailes religiosos de Tarapacá y Antofagasta. Una subcultura en vías de transformación integrativa. Tesis. FLACSO. Santiago.

_ 1973. Los santuarios de La Tirana, Ayquina y Las Peñas. Revista de la Universidad Técnica del Estado 11 y 12.

- 1980. Holocausto al progreso. Centro de Estudios y Documentación Latinoamericano, Amsterdam.

VARGAS, P., 1979. Isluga: Una problemática para el desarrollo. Tesis de Licenciatura en Antropología, Universidad de Chile, Santiago. 